\title{
Correlation between the sources of carbohydrate assimilation and spoilage of food items by pathogenic and non-pathogenic yeast
}

\author{
Mukta Singh, Karuna Singh, Divya and Aradhana Yadav
}

\begin{abstract}
Biologically active compounds are extra nutritional constituents that typically occur in small quantities in foods, vary widely in chemical structure and beneficially affect health and well-being. Nutritional yeast is the powerhouse of nutrients and it's a great addition to healthy life style especially for the vegetarians. Nutritional yeast is deactivated yeast, often a strain of Saccharomyces cerevisiae, which is sold commercially as a food product. Some of the sub species of Crytptococcus neoformans are pathogenic for the human immune system. Yeasts for obtaining energy undergo assimilation reaction i.e. break down of carbohydrate into its monomeric unit by various enzymatic activities produced by various yeasts.In the present study carbohydrate assimilation was studied by using different food products having pathogenic and non-pathogenic yeasts. All the17 isolates of pathogenic yeast were used and two sub species of S. cerevisiae and Rhodotorulla which were isolated from yeast powder and curd, respectively. It was seen by the use of spectrophotometer i.e. if the isolate has greater efficiency to assimilate food products then that food product having specific carbohydrate will be easily spoiled by the yeast. It was revealed by results that all the pathogenic yeast has high absorbance efficiency for fructose. It has been statistically proved ' $t$ ' calculated value (6.68) is greater than value of tabulated value (2.05) at 5\% level of significant. The Rhodotourla obtained from curd sample, have high absorbance value $(0.83)$ at is more favourable in food fermentation when food ingredients contains honey as energy. In milk proper absorbance value not obtained, indicating that Rhodotourla can be utilized in fermentation but not too much extent.
\end{abstract}

Key Words : Yeast, Carbohydrates, Assimilation, Pathogenic, Non pathogenic

How to cite this article : Singh, Mukta, Singh, Karuna, Divya and Yadav, Aradhana (2017). Correlation between the sources of carbohydrate assimilation and spoilage of food items by pathogenic and non-pathogenic yeast. Food Sci. Res. J., 8(2): 432-433, DOI : 10.15740/HAS/FSRJ/8.2/432-433. 